\title{
A WEAK TYPE INEQUALITY FOR CAUCHY TRANSFORMS OF FINITE MEASURES
}

\author{
JOAN VERDERA* \\ Dedicat a la memoiria d'en Pere Menal
}

Abstract

In this note we present a simple proof of a recent result of Mattila and Melnikov on the existence of $\lim _{\varepsilon \rightarrow 0} \int_{|\zeta-z|>\varepsilon}(\zeta-z)^{-1} d \mu(\zeta)$ for finite Bord measures $\mu$ in the plane.

\section{Introduction}

In this note we present a simple proof of an interesting recent result of Mattila and Melnikov [5] on the existence, in the principal value sense, of the Cauchy transform of a finite Borel measure. To be precise, given a complex finite Borel measure $\mu$ in the plane, set

$$
C \mu(z)=\lim _{\varepsilon \rightarrow 0} \int_{|\zeta-z|>\varepsilon}(\zeta-z)^{-1} d \mu(\zeta)
$$

whenever the limit exists. The main result of [5] is that, given a rectifable curve $\Gamma$, the limit in (1) exists almost everywhere with respect to arc length on $\Gamma$.

Since any rectifiable curve is the union of a set of zero length and a countable family of Lipschitz graphs, in proving the above statement one can assume that the curve under consideration is a Lipschitz graph. Now, if $\mu$ is supported on $\Gamma$ the result follows from the Coifman-MeyerMcIntosh [2] $L^{2}$ estimates for the Cauchy integral on Lipschitz graphs. Thus, the point of the Mattila-Melnikov Theoren is that there is no restraint whatsoever on the support of $\mu$.

*Partially supported by DCICYT (PB89-0311). 
The a.e. existence of the principal value (1) is a consequence, via standard arguments, of a weak type estimate which we now proceed to describe in the setting of Ahlfors-David regular curves, the most general class of rectifiable curves for which the Cauchy integral satisfies $L^{2}$ estimates [3]:

Let $\Gamma$ be a rectifiable curve and $\Lambda$ be one dimensional Hausdorff measure restricted to $\Gamma$. One says that $\Gamma$ is Ahlfors-David regular whenever

$$
\Lambda(\Delta(z, r) \cap \Gamma) \leq C r, \quad z \in \mathbb{C}
$$

for some constant $C$ and any disc $\Delta(z, r)$ with center $z$ and radius $r$.

Set

$$
C_{\varepsilon} \mu(z)=\int_{|\zeta-z|>\varepsilon}(\zeta-z)^{-1} d \mu(\zeta), \quad z \in \mathbb{C}, \quad \varepsilon>0
$$

and

$$
C^{*} \mu(z)=\sup _{\varepsilon>0}\left|C_{\varepsilon} \mu(z)\right| ; \quad z \in \mathbb{C}
$$

In [5] the following inequality was proved for Lipschitz, graphs.

Theorem. If $\Gamma$ is an Ahlfor's-David regular curve then

$$
\Lambda\left\{z \in \Gamma: C^{*} \mu(z)>t\right\} \leq C t^{-1}\|\mu\|
$$

for all complex finite Borel measures $\mu$ in the plane.

We proceed now to explain the main idea in our proof of (2). The interested reader will find complete details in Section 2.

The $L^{2}$ inequalities for $C^{*}$ proved by David, combined with standard Calderón-Zygmund Theory on homogeneous spaces, tell us that (2) is true when $\mu$ is supported on $\Gamma$. In particular, for measures $\mu$ supported on $\Gamma$, one has

$$
\Lambda\{z:|C \mu(z)|>t\} \leq C t^{-1}\|\mu\| .
$$

Now, a weak type inequality for a linear operator has a dual formulation (see Section 2) which, in the case of (3) for measures supported on $\Gamma$ turns out to be

$$
\Lambda(K) \leq C \sup \left\{\int b d \Lambda: b \in L^{\infty}(K), 0 \leq b \leq 1,|C(b d \Lambda)| \leq 1 \Lambda \text {-a.e. }\right\},
$$

for any compact subset $K$ of $\Gamma$. 
On the other hand (2) (without any restriction on the support of $\mu$ ) is equivalent to (3) (without any restriction on the support of $\mu$ ), because a suitable Cotlar type inequality is available in our setting, and (3) (without any restriction on the support of $\mu$ ) can be dualized to

$$
\begin{aligned}
& \Lambda(K) \leq C \sup \left\{\int b d \Lambda: b \in L^{\infty}(K), 0 \leq b \leq 1,\right. \\
& \qquad \text {-a.e., }|C(b d \Lambda)| \leq 1 \text { on } C \backslash \Gamma\},
\end{aligned}
$$

for any compact subset $K$ of $\Gamma$.

Therefore we are left with the task of showing that for $b \in L^{\infty}(K), K$ compact in $\Gamma,|b| \leq 1$ and $|C(b d \Lambda)| \leq 1 \Lambda$-a.e. imply $|C(b d \Lambda)| \leq C=$ $C(\Gamma)$ on $C \backslash \Gamma$, which turns out to be a simple matter $[1$, p. 110].

\section{Proof of the Theorem}

We start by showing how to dualize a weak type inequality for a linear operator.

Let $X$ and $Y$ be locally compact Hausdorff spaces, $\lambda$ a positive Radon measure on $X$ and $T$ a bounded linear operator from the space $M(X)$ of all complex finite Radon measures on $X$ into $C_{0}(Y)$, the space of continuous functions on $Y$ vanishing at $\infty$. Assume, furthermore, that the transpose of $T$, say $T^{*}$, sends boundedly $M(Y)$ into $C_{0}(X)$.

Lemma. The following statements are equivalent.

(i) $\lambda\left\{x:\left|T^{*} \mu(x)\right|>t\right\} \leq C t^{-1}\|\mu\|$

(ii) $\lambda(K) \leq C \sup \left\{\int b d \lambda: b \in L^{\infty}(K), 0 \leq b \leq 1 \lambda\right.$-a.e., $|T b| \leq 1$ on $Y\}$,

for any compact subset $K$ of $X$.

Moreover the least constants in (i) and (ii), say $c_{1}$ and $c_{2}$, satisfy $A^{-1} \leq c_{1} c_{2}^{-1} \leq A$, for some absolute constant $A$.

Proof: For (i) $\Rightarrow$ (ii) see [1, Th. 23, p. 107].

The reverse implication is very simple. It is clearly enough to prove that for each measure $\mu$ one has $\lambda\left\{x: R e T^{*} \mu(x)>1\right\} \leq C\|\mu\|$. Take a compact subset $K$ of $X$ such that $\operatorname{Re} T^{*} \mu>1$ on $K$ and the left hand side of the last incquality is not greater than twice $\lambda(K)$. By (ii), $\lambda(K) \leq C \int b d \lambda$, for some $b \in L^{\infty}(K), 0 \leq b \leq 1 \lambda$-a.c., $|T b| \leq 1$ on $Y$. Thus

$$
\int b d \lambda \leq \int b \operatorname{Re} T^{*} \mu d \lambda=\operatorname{Re} \int T b d \mu \leq\|\mu\|,
$$


which gives (i).

To apply the lemma it will be necessary to replace $C_{\varepsilon} \mu$ by another operator $K_{\varepsilon} \mu$, in such a way that $K_{\varepsilon} / \mu$ is a continuous furiction for each $\varepsilon$ and $\mu$. A convenient choice for $K_{\varepsilon} \mu$ is

$$
K_{\epsilon} \mu(z)=\int K_{\varepsilon}(\zeta-z) d \mu(\zeta)
$$

where $K_{\varepsilon}(z)$ is $1 / z$ for $|z| \geq \varepsilon$ and $\bar{z} / \epsilon^{2}$ for $|z| \leq \varepsilon$. In other words, $K_{\epsilon}=z^{-1} * \chi_{\epsilon}, \chi_{\epsilon}$ being the characteristic function of $\Delta(0, \varepsilon)$ divided by $\pi \varepsilon^{2}$. Thus $K_{\varepsilon}(z)$ is just a regularization of the kernel $1 / z$.

Set $M \mu(z)=\sup \left\{\mu \mid(\Delta(0, \varepsilon)) \varepsilon^{-1}\right.$. It is then clear that

$$
\left|C_{\varepsilon} \mu(z)-K_{\varepsilon} \mu(z)\right| \leq M \mu(z), \quad z \in \mathbb{C}
$$

Since $M$ satisfies the required weak type estimate and (2) holds for measures $\mu$ supported on $\Gamma$, because of [3], we get for such measures

$$
\Lambda\left\{z:\left|K_{\varepsilon} \mu *(z)\right|>t\right\} \leq C t^{-1}\|\mu\|
$$

with a constant $C$ independent of $\varepsilon$. Applying the Lemma to $X=$ $\Gamma, \lambda=\Lambda, Y=\Gamma, T=K_{\varepsilon}$, we conclude that given any compact $K \subset \Gamma$ we can find $b \in L^{\infty}(K), 0 \leq b \leq 1 \Lambda$-a.e., $\left|K_{\epsilon} b\right| \leq 1$ on $\Gamma$ such that $\Lambda(K) \leq C \int b d \Lambda$. It is now easy to ascertain as in $[1, \mathrm{p}$. 110] that, for some constant $C\left(\mathrm{I}^{\prime}\right)$ depending only on $\Gamma,\left|K_{\varepsilon} b\right| \leq C(\mathrm{\Gamma})$ on $\mathbb{C}$.

A second appeal to the Lemma for $X=\Gamma, \lambda=\Lambda, Y=C, T=K_{\varepsilon}$, gives (5) for all finite Borel measures $\mu$ and some constant $C$ independent, of $\varepsilon$.

To complete the proof we only need to apply a well known technology. The subspace of $M(\mathbb{C})$ defined by $S=\left\{\varphi(z) d z_{\mathrm{r}}+\nu\right\}$, where $\varphi \in C_{0}^{\infty}(\mathbb{C})$ and $\nu$ is supported on a closed set of vanishing $\Lambda$-measure, is norm dense in $M(\mathbb{C})$, and clearly $C \mu(z)$ exists $\Lambda$-a.e. for $\mu \in S$. Since (5) holds with $K_{\varepsilon}$ replaced by $C_{\varepsilon}$ because of (4), $C$ extends from $S$ to an operator, which we still call $C$, defined on $M(\mathbb{C})$ and satisfying (3) for each $\mu \in M(\mathbb{C})$. Now one proves, exactly as in $[4$, p. 56] that for each $p, 0<p<1$, there exists a constant $C_{p}$ with

$$
C^{*} \mu(z) \leq C_{y} M \mu(z)+C_{p} M\left(|C \mu|^{p}\right)^{1 / p}(z)
$$

Kolmogorov's inequality $[4$, p. 5$]$ and (6) now give (2): 


\section{Final remarks}

The history of dualizing a weak type inequality, as far as the author knows, is as follows. It, appears for the first time in a paper by N. X. Uy (Removable sets of analytic functions satisfying a Lipschitz condition, Ark. Mat. 17 (1979), 19-27), where it was used to characterize the removable sets for Lipschitz cualytic functions in the plane as those having zero area. $S$. Hruscev, who had also been working in some removability problems for analytic functions (The problem of simultaneous approximation and removal of singularities of Cauchy-type integrals, Proceedings of the Steklov Inst. of Malh 4 (1979), 133-203) found a neat proof of Uy's result which was reported in a paper by the anthor $\left(C^{n}\right.$ approximation by solutions of elliptic equations, and Calderón-Zygmund operators, Duke Math. J. 55 (1987), 155-187). Davie and Oksendal gave essentially the proof of the Lemma in Section 2 while proving that the Denjoy conjecture follows from Calderón $L^{2}$ estimates for the Cauchy integral on Lipschit/ graphs with snall Lipschitz constant (see their joint paper Analytic capacity and differentiability properties of finely hamonic functions, Acla Math. 149 (1982), 127-152). More recently Murai has used dual versions of woak type inequalities to relate analytic capacity and length on chord-arc curves ("A real variable method for the Cauchy transform and analytic capacity", Lecture Notes in Math. 1307, Springer-Verlag, New York, 1988).

Finally, we would like to point out a couple of unsolved problems involving analytic capacity.

Let $m$ be a locally finite positive Borel measure in the plane. One would like to show that an inequality of the type .

$$
m\left\{z: C^{*} \mu(z)>t\right\} \leq C t^{-1}\|\mu\|
$$

is equivalent to

$$
m(K) \leq C \gamma(K), \text { for all compact sets } K,
$$

where $y$ is analytic capacity. Thus one is lead to ask whether or not an absolute constant $A$ can be found such that,

$$
\gamma\left\{z: C^{*} \mu(z)>t\right\} \leq A t^{-1}\|\mu\|,
$$

for all finite Borel measures $\mu$ in the plane.

If (7) were trues, then many aspects of the classical potential theory of the Riesz kernels would have their counterpart for the complex potential $1 / z$. 


\section{References}

1. M. CHRIST, "Lectures on singular integral operators," Regional Conference Series in Math. 77, Amer. Math. Soc., 1990.

2. R. R. COIFman, A. MCINTOSH AND Y. MEYER, L'integrale de Cauchy définit un opérateur borné sur $L^{2}$ sur les courbes Lipschitziennes, Ann. of Moth. 116 (1982), 361-388.

3. G. DAVID, Opérateurs integranx singuliers sur certaines courbes du plan complexe, Ann. Sci. Ec. Norm. Sup. 17 (1984), 157-189.

4. J. L. JouRNÉ, "Calderón-Zygmund operators, pseudo-differential operators and the Cauchy integral of Calderón," Lecture Notes in Math. 994, Springer-Verlag; Berlin, 1983.

5. P. MatTila and M. Melnikov, Existence and weak type inequalities for Cauchy integrals of general measires on rectifiable curves and sets, to appear in Proc. Amer. Math. Soc.

Departament de Matemàtiques Universitat Autònoma de Barcelona 08193 Bellaierra (Barcelona)

SPAIN

Rebut el 14 d'Octubre de 1991 